Letter to the Editor

\title{
Relationships between anhedonia and depressive symptoms in major depressive disorder
}

\author{
G Loas ${ }^{1}$, P Boyer ${ }^{2}$ \\ 'Service Hospitaio-Universitaire (Pr J Delahousse), Hôpital Pinel, Amiens, 80001 Cedex; \\ ${ }^{2}$ INSERM, 100, rue de la santé, 75674 Paris Cedex 14, France
}

Anhedonia, the lowered ability to experience pleasure, is an important symptom for the definition of depressive disorders, namely for the endogeneous or melancholic subtypes. In a previous work Loas et al (1992), using an operational definition of anhedonia, based on two reliable rating scales (Physical Anhedonia Scale, PAS, Chapman et al, 1976; Fawcett-Clark Pleasure Capacity Scale, FCPCS, Fawcett et al, 1983), we have shown that patients meeting criteria for a major depressive episode compared to controls, were more anhedonic. On the other hand, anhedonia seems to be a marker of depressive severity since there was a positive correlation $(r=0.33, P=0.009)$ between PAS score and the Hamilton Depression rating scale (HDRS).

Dr Snaith (University of Leeds), in reaction to this work, has suggested that it would be interesting to study the relationships between the anhedonia scales and each item of the HDRS. This work was conducted according to his suggestion.

The characteristics of the subjects have been previously mentioned (see Loas et al, 1992). Sixtyone inpatients ( 15 males, 46 females; mean age = $43.9, s d=12$ ) meeting the RDC criteria for primary major depressive disorder completed the PAS and the FCPCS. The depressive symptomatology was evaluated by the HDRS (17 items form). The subjects were evaluated during the first few days of their hospitalization and were undergoing treatment. The correlations (Pearson correlation coefficient) between both scores at anhedonia scales and the HDRS items are given in table I (only the statistically significant correlations are indicated: $P$ $<0.05$ ). Four significant correlations can be evidenced for PAS and one for FCPCS. PAS score correlates significantly with the following HDRS items: retardation, anxiety (psychic), suicide and somatic symptom (gastro-intestinal). The link between anhedonia and psychomotor retardation has already been found by Pogue-Geile and Harrow (1984) in major depression and schizophrenia and by Kulhara and Chadda (1987) in schizophrenia. The relationship between anhedonia and suicide was suggested by Watson and Kucala (1978). More recently Fawcett et al (1990) have shown that anhedonia was associated with suicide within one year in major affective disorders and suggests that effective pharmacotherapy and cognitive therapy could modify the influence of this risk factor.

It would be of interest to use PAS as a measure of anhedonia in depressed patients. This self-rating scale is easy to fill out and could predict, in the case of elevated scores, an imminent suicide attempt, emphasizing the usefulness of the guidelines suggested by Fawcett.

Table I. Intercorrelations between PAS, FCPCS and HDRS items in patients presenting primary major depression $(P<0.05$ for
all the values).

\begin{tabular}{lcc}
\hline HDRS & PAS & FCPCS \\
\hline Suicide & 0.28 & \\
Retardation & 0.33 & \\
Anxiety psychic & 0.31 & \\
Somatic gastro-intestinal & 0.26 & \\
Somatic general & & $(-) 0.29$ \\
\hline
\end{tabular}




\section{References}

Chapman LJ, Chapman JP, Raulin ML (1976) Scales for physical and social anhedonia. J Abnorm Psychol 4, 374-382

Fawcett J, Clark DC, Scheftner WA, Gibbons RD (1983) Assessing anhedonia in psychiatric patients; the pleasure scale. Arch Gen Psychiatry 40, 79-84

Fawcett J, Scheftner WA, Fogg L, Clark DC, Young MA, Hedeker D, Gibbons R (1990), Time-related predictors of suicide in major affective disorder. Am J Psychiatry $147,9,1189-1194$
Kulhara P and Chadda R (1987) A study of negative symptoms in schizophrenia and depression. Comp Psychiatr 28, 3, 229-235

Loas G, Salinas E, Guelfi JD and SamuelLajeunesse B (1992) Physical anhedonia in major depressive disorder. J Affect Disord 25, 139146

Pogue-Geile MF, Harrow M (1984) Negative and positive symptoms in schizophrenia and depression: a follow up. Schizo Bull 10, 3, 371-387

Watson CG, Kucala T (1978) Anhedonia and death. Psychol Rep 43, 1120-1122 